Article

\title{
Evaluating the Age-Dependent Potential for Protein Deposition in Naked Neck Meat Type Chicken
}

\author{
Daulat R. Khan ${ }^{1}$, Christian Wecke ${ }^{1}$, Ahmad R. Sharifi ${ }^{2}$ and Frank Liebert ${ }^{1, *}$ \\ ${ }^{1}$ Division Animal Nutrition Physiology, Department of Animal Sciences, Georg-August-University, \\ Kellnerweg 6, 37077 Goettingen, Germany; E-Mails: dkhan@gwdg.de (D.R.K.); \\ cwecke@gwdg.de (C.W.) \\ ${ }^{2}$ Division Animal Breeding and Genetics, Department of Animal Sciences, Georg-August-University, \\ Albrecht-Thaer-Weg 3, 37075 Goettingen, Germany; E-Mail: rsharif@gwdg.de \\ * Author to whom correspondence should be addressed; E-Mail: flieber@ gwdg.de; \\ Tel.: +49-551-39-33331; Fax: +49-551-39-33343.
}

Academic Editor: Clive J. C. Phillips

Received: 29 September 2014 / Accepted: 9 January 2015 / Published: 19 January 2015

Simple Summary: Growth rates of fast-growing chickens are reduced by a higher ambient temperature (AT) because of difficulties in dissipating heat through the feather coverage. Naked neck meat type genotypes could be helpful in increasing the tolerance for high AT. However, basic model parameters of this genotype necessary to further assess amino acid requirements are as yet unavailable. The experiments were conducted to estimate both the daily nitrogen maintenance requirement (NMR) and the potential for daily nitrogen retention $\mathrm{NR}_{\max } \mathrm{T}$ ). These observed model parameters provide the basic information to characterize the growth potential of the genotype for further application in modeling of individual amino acid requirements of naked neck meat type chicken.

\begin{abstract}
The introduction of the naked neck gene $(\mathrm{Na})$ into modern meat type chicken is known to be helpful in increasing the tolerance for a high ambient temperature (AT) by reducing the feather coverage which allows for a higher level of heat dissipation compared to normally feathered (na/na) birds. In addition, reduced feather coverage could affect requirements for sulfur containing amino acids. As a prerequisite for further modeling of individual amino acid requirements, the daily $\mathrm{N}$ maintenance requirement (NMR) and the threshold value of daily $\mathrm{N}$ retention $\left(\mathrm{NR}_{\max } \mathrm{T}\right)$ were determined. This was carried out using graded dietary protein supply and exponential modeling between $\mathrm{N}$ intake $(\mathrm{NI})$ and $\mathrm{N}$ excretion (NEX) or N deposition (ND), respectively. Studies with homozygous ( $\mathrm{Na} / \mathrm{Na}$ ) and
\end{abstract}


heterozygous (Na/na) naked neck meat type chicken utilized 144 birds of average weight (50\% of each genotype and sex) within two $\mathrm{N}$ balance experiments during both the starter (days 10-20) and the grower period (days 25-35). Birds were randomly allotted to five diets with graded dietary protein supply but constant protein quality. The observed estimates depending on genotype, sex and age varied for NMR and $\mathrm{NR}_{\max } \mathrm{T}$ from 224 to 395 and 2881 to $4049 \mathrm{mg} \mathrm{N} / \mathrm{BW}_{\mathrm{kg}}{ }^{0.67} /$ day, respectively.

Keywords: naked neck meat type chicken; $\mathrm{N}$ utilization model; $\mathrm{N}$ maintenance requirement; protein deposition potential

\section{Introduction}

In the last few decades, substantial genetic progress has been made in broiler growth, feed efficiency, meat yield and increased metabolic rate [1,2]. Conversely, the birds became more sensitive to suboptimal environmental conditions, especially to hot ambient condition [3]. Compared with muscle growth, the cardiovascular and respiratory systems do not adapt as well to extreme environment conditions, which resulted in a lower capability to regulate energy expenditure and the body water balance system [2]. There are two different main strategies for improving the tolerance of chicken for higher ambient temperatures (AT). Firstly, by reducing temperatures through convection, conduction, radiation and by maintaining house temperature using different management practices, or by decreasing heat production [4]. However, some factors may lead to higher production costs [5]. Secondly, through the introduction of breeds well adapted to high AT and also showing a higher growth potential. Such a breeding and selection strategy can be particularly effective to achieve improved results in unfavorable tropical environments. A useful breeding strategy is to create a breeding stock carrying a major gene for better heat tolerance. The naked neck dominant gene $(\mathrm{Na})$ is known to improve heat endurance through different pathways: Either by decreasing the metabolic heat increment or by diminishing the insulating power of bird's plumage which leads to higher heat loss and thus ultimately helps in regulating body temperature [4]. Previous studies investigated the crude protein (CP) requirement for naked neck birds, but furnished only uncertain information. According to the high protein content of feathers, Ajang et al. [6] suggested that the degree of feathering in fast and slow feathering broilers may influence the $\mathrm{CP}$ requirement of chicken. Yalcin et al. [7] observed that naked neck birds did not require less dietary protein because of their reduced feather covering. However, conclusive amino acid (AA) requirement studies have not been carried out for the naked neck birds.

Further applications of an exponential modeling procedure [8-13] were utilized to fill this gap. This study requires experimental data regarding both the age dependent potential for nitrogen deposition and the daily nitrogen maintenance requirement of this particular genotype. It was the aim of the study to predict these model parameters for homozygous and heterozygous ( $\mathrm{Na} / \mathrm{Na}$; $\mathrm{Na} / \mathrm{na})$ naked neck meat type chicken depending on age and sex. 


\section{Experimental Section}

\subsection{Stock and Husbandry}

The experiments were conducted at the Division of Animal Nutrition Physiology, Department of Animal Sciences at the Georg-August-University Goettingen with the approval of the Animal Welfare Law Committee of Lower Saxony, Germany.

Heterozygous parent stock (Na/na) from a heavy broiler sire line (Aviagen ${ }^{\circledR}$ Poultry Breeders UK) with a genetic disposition for high growth performance was used for obtaining desired experimental homozygous ( $\mathrm{Na} / \mathrm{Na}$ ); and heterozygous ( $\mathrm{Na} / \mathrm{na}$ ) chicks. The only $\mathrm{Na} / \mathrm{Na}$ and $\mathrm{Na} / \mathrm{na}$ genotypes used in the present studies were the full sib and half sib offspring of heterozygous ( $\mathrm{Na} / \mathrm{na}$ ) naked neck parents. These share the same genetic background to a high degree, which facilitated the accurate measurement of $\mathrm{N}$ deposition for both the genetics during starter and grower period.

All of the experimental birds were reared under standardized housing and feeding regimes from day 0 to 5 and 0 to 20. Afterwards, a total of 144 birds of average weight (72 $\mathrm{Na} / \mathrm{Na}$ and $72 \mathrm{Na} / \mathrm{na}$, each $50 \%$ male and female) were selected for $\mathrm{N}$ balance experiments involving both starter (days 10-20; $2 \times 36$ birds) and grower period (days 25-35; $2 \times 36$ birds). The birds were individually housed in metabolic cages with wire floors, individual feeders and self-drinking systems. The room temperature was gradually reduced from $32{ }^{\circ} \mathrm{C}$ to $23{ }^{\circ} \mathrm{C}$ with increasing age. Humidity was maintained between $60 \%-70 \%$ and monochromatic light was provided for $23 \mathrm{~h}$ following $1 \mathrm{~h}$ darkness.

\subsection{Feeding and Sampling}

Chickens of both genotypes and sexes were randomly allotted to five experimental diets (N1-N5). The formulation of the pelleted diets was based on a constant mixture of the native feed protein sources of soy protein concentrate (SPC), wheat, maize and wheat gluten, but diluted with wheat starch to create graded crude protein (CP) levels between $10 \%$ and 38\% (Tables 1 and 2). For future applications of the data, methionine (Met) was set as first limiting amino acid (LAA) to calculate requirement data following principles of the diet dilution technique. Except Met, other amino acid (AA) requirements were fulfilled according to NRC [14] recommendations and the achieved dietary amino acid pattern was kept unchanged independently of dietary protein levels at both the age periods (Table 2). According to age dependent energy demands, the energy content of the grower diets was enhanced and the CP contents were slightly decreased without changing the AA profile. The dietary $\mathrm{Ca}$ and $\mathrm{P}$ supply was $1.0 \%$ and $0.6 \%$ in starter and $0.85 \%$ and $0.55 \%$ in grower feed, respectively. Further mineral and vitamin supplementations were in line with current recommendations $[14,15]$.

Two experimental periods, i.e., starter (days 10-20) and grower period (days 25-35) were conducted for every genotype and sex. The individual experimental period was divided into an adaptation period (5 days) and two consecutive quantitative excreta collecting periods (each 5 days). At the beginning of the adaptation period, feed was given ad libitum to estimate the adequate level of individual feed intake under housing conditions in the metabolic cages. The individual feed supply was kept constant from day 3 of the adaptation period, slightly adapted during the first two days of the collecting period, and kept constant again up to the end of the collecting periods, respectively. To avoid effects of feed 
rejection by individual birds due to the extreme $\mathrm{CP}$ contents, $\mathrm{N}$ balance studies were conducted with 10 and 8 replicates for the lowest (N1) and highest CP diets (N5) and 6 replicates (diets N2-4) per genotype, sex and age period, respectively. Excreta collection was conducted twice a day to prevent ammonia loss from non-acidified excreta. Excreta samples were immediately frozen $\left(-20^{\circ} \mathrm{C}\right)$ for further analysis.

Table 1. Composition of experimental diets (percentage as fed).

\begin{tabular}{|c|c|c|c|c|c|c|c|c|c|c|}
\hline \multirow[t]{2}{*}{ Ingredients } & \multicolumn{9}{|c|}{ Diets } & \multirow[b]{3}{*}{ N5 } \\
\hline & \multicolumn{5}{|c|}{ Starter (Days 10-20) } & \multicolumn{4}{|c|}{ Grower (Days 25-35) } & \\
\hline Diet $^{1}$ & N1 & $\mathbf{N 2}$ & N3 & N4 & N5 & N1 & $\mathbf{N 2}$ & N3 & N4 & \\
\hline Maize & 6.62 & 10.59 & 14.56 & 18.45 & 22.50 & 5.95 & 9.70 & 13.67 & 18.08 & 22.05 \\
\hline Wheat & 5.09 & 8.14 & 11.19 & 14.19 & 17.30 & 4.58 & 7.46 & 10.51 & 13.90 & 16.95 \\
\hline Soy protein concentrate & 10.15 & 16.23 & 22.32 & 28.29 & 34.50 & 9.13 & 14.88 & 20.96 & 27.72 & 33.81 \\
\hline Fish meal & 1.91 & 3.06 & 4.21 & 5.33 & 6.50 & 1.72 & 2.80 & 3.95 & 5.22 & 6.37 \\
\hline Wheat gluten & 1.76 & 2.82 & 3.88 & 4.92 & 6.00 & 1.59 & 2.59 & 3.65 & 4.82 & 5.88 \\
\hline Soybean oil & 3.09 & 4.94 & 6.79 & 8.61 & 10.50 & 3.24 & 5.28 & 7.44 & 9.84 & 12.00 \\
\hline Cellulose & 1.80 & 1.35 & 0.90 & 0.46 & - & 1.82 & 1.40 & 0.95 & 0.45 & - \\
\hline Wheat starch & 65.54 & 49.17 & 32.78 & 16.72 & - & 68.42 & 52.70 & 35.99 & 17.45 & 0.78 \\
\hline Premix ${ }^{2}$ & 1.00 & 1.00 & 1.00 & 1.00 & 1.00 & 1.00 & 1.00 & 1.00 & 1.00 & 1.00 \\
\hline DCP & 2.50 & 2.08 & 1.65 & 1.25 & 0.80 & 2.30 & 1.89 & 1.45 & 0.97 & 0.55 \\
\hline $\mathrm{CaCO}_{3}$ & 0.30 & 0.40 & 0.52 & 0.60 & 0.74 & 0.06 & 0.15 & 0.28 & 0.41 & 0.50 \\
\hline $\mathrm{NaCl}$ & 0.24 & 0.22 & 0.20 & 0.18 & 0.16 & 0.19 & 0.15 & 0.15 & 0.12 & 0.10 \\
\hline
\end{tabular}

\footnotetext{
${ }^{1}$ Starter $=\mathrm{N} 1: 10.85 ; \mathrm{N} 2: 17.44 ; \mathrm{N} 3: 24.10 ; \mathrm{N} 4: 30.70 ; \mathrm{N} 5: 37.63 \%$ CP on DM basis; grower = N1: 9.75; N2: 15.96; N3: 22.59; N4:30.05; N5:36.83percent crude protein on dry matter basis. ${ }^{2}$ Provided (per kilogram of diet): Vitamin A, 12,000 IU; vitamin $\mathrm{D}_{3}, 3,500 \mathrm{IU}$; vitamin $\mathrm{E}, 40 \mathrm{mg}$; thiamin, $2.5 \mathrm{mg}$; riboflavin, $8.0 \mathrm{mg}$; vitamin $\mathrm{B}_{6}, 6.0 \mathrm{mg}$; vitamin $\mathrm{B}_{12}, 32 \mu \mathrm{g}$; vitamin $\mathrm{K}_{3}, 4.5 \mathrm{mg}$; nicotinic acid, $45 \mathrm{mg}$; $\mathrm{CaCO}_{3}, 15 \mathrm{mg}$; folic acid, $1.2 \mathrm{mg}$; biotin, $50 \mu \mathrm{g}$; choline chloride, $550 \mathrm{mg}$; Mn, $100 \mathrm{mg}$; Zn, $80 \mathrm{mg}$; Fe, $30 \mathrm{mg}$; Cu, $20 \mathrm{mg}$; I, $1.2 \mathrm{mg}$; Co, $0.4 \mathrm{mg}$; Se, $0.4 \mathrm{mg}$; and butylated hydroxytoluene, $100 \mathrm{mg}$.
}

Table 2. Analyzed nutrient content and amino acid composition of experimental diets (percent on dry matter basis).

\begin{tabular}{|c|c|c|c|c|c|c|c|c|c|c|}
\hline \multirow{3}{*}{$\begin{array}{c}\text { Nutrients } \\
\text { Diet }\end{array}$} & \multicolumn{9}{|c|}{ Diets } & \multirow[b]{3}{*}{ N5 } \\
\hline & \multicolumn{5}{|c|}{ Starter (Days 10-20) } & \multicolumn{4}{|c|}{ Grower (Days 25-35) } & \\
\hline & N1 & $\mathbf{N 2}$ & N3 & N4 & N5 & N1 & $\mathbf{N 2}$ & N3 & N4 & \\
\hline Crude protein & 10.85 & 17.44 & 24.10 & 30.70 & 37.63 & 9.75 & 15.96 & 22.59 & 30.05 & 36.83 \\
\hline Ether extract & 3.94 & 6.26 & 8.62 & 10.96 & 13.41 & 4.04 & 6.55 & 9.24 & 12.25 & 14.99 \\
\hline Crude fiber & 2.35 & 2.36 & 2.37 & 2.39 & 2.40 & 2.30 & 2.31 & 2.33 & 2.34 & 2.35 \\
\hline Crude ash & 5.43 & 5.86 & 6.32 & 6.75 & 7.24 & 4.78 & 5.14 & 5.62 & 6.11 & 6.55 \\
\hline $\mathrm{N}$-free extract & 70.69 & 60.59 & 50.13 & 40.87 & 30.40 & 72.48 & 62.95 & 52.67 & 41.18 & 30.75 \\
\hline Starch & 65.02 & 55.65 & 46.17 & 36.78 & 26.89 & 70.35 & 60.04 & 49.75 & 37.58 & 27.16 \\
\hline Total sugars & 1.64 & 1.64 & 1.64 & 1.63 & 1.63 & 1.74 & 1.70 & 1.69 & 1.64 & 1.61 \\
\hline $\operatorname{AMEn}^{1}(\mathrm{MJ} / \mathrm{kg})$ & 14.73 & 15.5 & 15.33 & 15.16 & 15.37 & 15.02 & 15.21 & 15.43 & 15.47 & 15.59 \\
\hline Amino Acids & \multicolumn{5}{|c|}{ Amino Acid Composition (g/100g Crude Protein) } & \multicolumn{4}{|c|}{ Amino Acid Ratio (Lys = 100) } & \\
\hline Lys & \multicolumn{5}{|c|}{5.09} & \multicolumn{4}{|c|}{100} & \\
\hline Met & \multicolumn{5}{|c|}{1.44} & \multicolumn{4}{|c|}{28} & \\
\hline
\end{tabular}


Table 2. Cont.

\begin{tabular}{ccc}
\hline Nutrients & \multicolumn{2}{c}{ Diets } \\
\hline Amino Acids & Starter (Days 10-20) & Grower (Days 25-35) \\
\hline Cys & 1.46 & Amino Acid Ratio (Lys = 100) \\
Thr & 3.63 & 29 \\
Trp & 0.96 & 71 \\
Arg & 6.26 & 19 \\
Ile & 4.03 & 123 \\
Leu & 7.50 & 79 \\
Val & 4.24 & 147 \\
Phe & 4.71 & 83 \\
\hline
\end{tabular}

${ }^{1}$ Nitrogen corrected apparent metabolizable energy, calculated according to WPSA [16].

\subsection{Laboratory Analysis}

Dietary ingredients, experimental diets and excreta were analyzed according to the German standards [17]. The $\mathrm{N}$ content was quantified using to the Dumas method (Leco ${ }^{\circledR}$ LP-2000, Leco ${ }^{\circledR}$ Instrument $\mathrm{GmbH}$, Kirchheim, Germany) and $\mathrm{CP}$ was calculated with factor 6.25. Amino acids (AA) of the protein sources were analyzed by ion-exchange chromatography (Biochrom ${ }^{\circledR} 30$, Biochrom Ltd. Cambridge, UK) following acid hydrolysis with and without an oxidation step for quantitative determination of sulfur-containing amino acids. Ether extract was analyzed following $\mathrm{HCl}$ hydrolysis of the feed samples.

\subsection{Model Parameter Assessment and Statistics}

Model applications basically require actual information regarding the daily $\mathrm{N}$ maintenance requirement $(\mathrm{NMR})$ and the threshold value for daily $\mathrm{N}$ retention $\left(\mathrm{NR}_{\max } \mathrm{T}\right)$. The $\mathrm{NMR}$ estimation utilized the exponential regression between $\mathrm{N}$ intake (NI) and daily $\mathrm{N}$ excretion (NEX) following graded dietary protein supply [8-11,18-20] in order to prevent non-physiological $\mathrm{N}$ free feeding conditions. The obtained NMR value was set as point of intersection with the y-axis. Accordingly, estimating of threshold value $\mathrm{NR}_{\max } \mathrm{T}$ utilized the regression of $\mathrm{N}$ deposition (ND) depending on NI:

$$
\begin{gathered}
\mathrm{NR}=\mathrm{NR}_{\max } \mathrm{T}\left(1-\mathrm{e}^{-\mathrm{bNI}}\right) \\
\mathrm{ND}=\mathrm{NR}_{\max } \mathrm{T}\left(1-\mathrm{e}^{-\mathrm{bNI}}\right)-\mathrm{NMR}
\end{gathered}
$$

where $\mathrm{NR}=$ daily $\mathrm{N}$ retention $\left(\mathrm{ND}+\mathrm{NMR} ; \mathrm{mg} / \mathrm{BW}_{\mathrm{kg}}{ }^{0.67}\right) ; \mathrm{NR}_{\max } \mathrm{T}=$ theoretical maximum for daily $\mathrm{NR}\left(\mathrm{mg} / \mathrm{BW}_{\mathrm{kg}}{ }^{0.67}\right) ; \mathrm{ND}=$ daily $\mathrm{N}$ deposition $\left(\mathrm{mg} / \mathrm{BW}_{\mathrm{kg}}{ }^{0.67}\right) ; \mathrm{NMR}=$ daily nitrogen maintenance requirement $\left(\mathrm{mg} / \mathrm{BW}_{\mathrm{kg}}{ }^{0.67}\right) ; \mathrm{NI}=$ daily $\mathrm{N}$ intake $\left(\mathrm{mg} / \mathrm{BW}_{\mathrm{kg}}{ }^{0.67}\right)$; e = basic number of natural logarithm (ln); $b=$ slope of the $\mathrm{N}$ retention curve (parameter of feed protein quality).

The model parameter $b$ as an indicator of the dietary protein quality was derived following logarithmization and transformation of Equation (1):

$$
b=\left[\ln N R_{\max } \mathrm{T}-\ln \left(\mathrm{NR}_{\max } \mathrm{T}-\mathrm{NR}\right)\right] / \mathrm{NI}
$$


where $b=$ model parameter for dietary protein quality; $\ln =$ natural logarithm; $\mathrm{NR}_{\max } \mathrm{T}=$ theoretical maximum for daily $\mathrm{NR} ; \mathrm{NR}=$ nitrogen retention; $\mathrm{NI}=$ nitrogen intake.

Further details of this model application for evaluating the dietary protein quality independent on varying NI are reported elsewhere [13,19,21].

$\mathrm{ND}_{\max } \mathrm{T}$ as the theoretical maximum for $\mathrm{N}$ deposition $\left(\mathrm{ND}_{\max } \mathrm{T}+\mathrm{NMR}=\mathrm{NR}_{\max } \mathrm{T}\right)$ was estimated following several iteration steps by the Levenberg-Marquardt algorithm within the SPSS statistical package. The attribute "theoretical" (T) indicates that the estimated threshold value is not in the area of practical growth data, but they characterize the estimated genetic potential that is not attainable due to several limitations in dietary and environmental factors. An overview about the applied modeling approach is given by Liebert [21].

Statistical analyses were run with SPSS. N balance data were analyzed by GLM ANOVA using the GLM procedure of SPSS software package (version 19.0 for Windows, IBM SPSS Statistics, Inc., Chicago, IL, USA). A multifactorial ANOVA was utilized using the main factors diet, genotypes, sex and age period. However, the only two-way interaction between selected variables revealed significant effects therefore the three and four way interactions were excluded. The comparison of means was carried out using LS-Means statements and adjusted by Tukey's test. Statistical analysis was performed according to the following model including main factors and significant interaction:

$$
\mathrm{y}_{\mathrm{ijklm}}=\mu+\mathrm{D}_{\mathrm{i}}+\mathrm{G}_{\mathrm{j}}+\mathrm{S}_{\mathrm{k}}+\mathrm{A}_{\mathrm{l}}+\mathrm{DG}_{\mathrm{ij}}+\mathrm{DS}_{\mathrm{ik}}+\mathrm{DA}_{\mathrm{il}}+\mathrm{GS}_{\mathrm{jk}}+\mathrm{GA}_{\mathrm{jl}}+\mathrm{SA}_{\mathrm{kl}}+\mathrm{e}_{\mathrm{ijklm}}
$$

where $\mathrm{y}_{i j k l m}$ is the dependent variable; $\mu$ is the general mean; $\mathrm{D}_{i}$ is the main effect of diet ( $\mathrm{i}=\mathrm{N} 1$ $\ldots \mathrm{N} 5$ ); $\mathrm{G}_{j}$ is the main effect of genotype (Na/Navs. Na/na); $\mathrm{S}_{k}$ is the main effect of sex (male $v s$. female); $\mathrm{A}_{l}$ is the main effect of age (starter vs. grower); DG, DS, DA, GA, GS and SA are the two-way interaction between the main effects and $\mathrm{e}_{i j k l m}$ is the random error.

\section{Results and Discussion}

Results of the analysis of variance involving all diets, genotypes, sexes and age periods and their two-way interactions are given in Tables 3. There was no significant main effect of genotypes. The genotypes' interactions with age period and sex revealed non-significant differences for all $\mathrm{N}$ balance parameters, mean BW and DM intake. However, interactions of genotype with the experimental diets (D) showed significant differences in NEX for different diets across genotype. Sex as a main effect did not indicate any significant effects for BW, DMI and NI. However, NEX and ND were significantly different between male and female chickens. Significant interaction of sex with the diet resulted in a significant difference for NI and ND. Interaction of sex with age periods revealed significant differences for MBW, DMI, NI and NEX except ND.

The average data from $\mathrm{N}$ balance studies depending on diet, genotype, sex and age period are summarized in 4 and 5. MBW and DMI in starter period (d 10-20) were significantly $(p<0.001)$ lower in the diet with lowest CP level (N1) than in diets N2-N5 with increased CP levels in male and female chickens of both genotypes (Table 4). According to earlier studies [8,9,11,13,20], NI and NEX increased significantly with increasing $\mathrm{CP}$ level in the experimental diets. ND for diet N1 was significantly lower compared to diets with higher CP contents. Diets N3-N5 yielded non-significant differences for ND among them. 
Table 3. Results derived from multi-factorial analysis of variance for all genotypes, sexes and age periods.

\begin{tabular}{|c|c|c|c|c|c|c|c|c|c|c|}
\hline & Diet & Geno-Type & Sex & Age Period & Diet $\times$ Genotype & Diet $\times$ Sex & Diet $\times$ Age & Genotype $\times$ Sex & Genotype $\times$ Age & Sex $\times$ Age \\
\hline & \multicolumn{10}{|c|}{ Probabilities } \\
\hline BW (g) & $<0.001$ & 0.633 & 0.274 & $<0.001$ & 0.956 & 0.945 & 0.002 & 0.432 & 0.547 & $<0.001$ \\
\hline DMI (g/d) & $<0.001$ & 0.827 & 0.559 & $<0.001$ & 0.985 & 0.882 & 0.001 & 0.327 & 0.578 & $<0.001$ \\
\hline $\mathrm{NI}^{1}$ & $<0.001$ & 0.844 & 0.055 & $<0.001$ & 0.579 & 0.001 & $<0.001$ & 0.635 & 0.952 & 0.002 \\
\hline $\mathrm{NEX}^{1}$ & $<0.001$ & 0.113 & $<0.001$ & $<0.001$ & 0.002 & 0.120 & $<0.001$ & 0.103 & 0.384 & 0.004 \\
\hline $\mathrm{ND}^{1}$ & $<0.001$ & 0.079 & $<0.001$ & $<0.001$ & 0.552 & 0.007 & $<0.001$ & 0.030 & 0.366 & 0.194 \\
\hline$b$-value ${ }^{2}$ & $<0.001$ & $<0.001$ & $<0.001$ & $<0.001$ & 0.328 & 0.950 & 0.017 & 0.183 & 0.914 & 0.001 \\
\hline
\end{tabular}

$\mathrm{BW}=$ Body weight, DMI = Dry matter intake, $\mathrm{NI}=\mathrm{N}$ intake, NEX = N excretion, ND = N deposition (NI-NEX). ${ }^{1} \mathrm{mg} / \mathrm{BW}_{\mathrm{kg}}{ }^{0.67} \mathrm{per} \mathrm{day}$

${ }^{2} b$-value $=$ Model parameter indicating protein quality $\left(b \cdot 10^{6}\right)$. 
Table 4. Results of $\mathrm{N}$ balance experiments depending on dietary protein content, genotype and sex during starter period (days 10-20) ${ }^{1}$.

\begin{tabular}{|c|c|c|c|c|c|}
\hline \multirow[t]{2}{*}{ Item } & \multicolumn{5}{|c|}{ Diet (Crude Protein as \% of Dry Matter) } \\
\hline & N1 (10.8) & N2 (17.4) & N3 (24.1) & N4 (30.7) & N5 (37.6) \\
\hline & \multicolumn{5}{|c|}{ Males $(\mathrm{Na} / \mathrm{Na})$} \\
\hline BW (g) & $152^{\mathrm{a}} \pm 7$ & $312^{\mathrm{b}} \pm 40$ & $381^{\mathrm{b}} \pm 58$ & $375^{\mathrm{b}} \pm 74$ & $389^{\mathrm{b}} \pm 46$ \\
\hline DMI $(\mathrm{g} / \mathrm{d})$ & $17.9^{\mathrm{a}} \pm 1.1$ & $47.1^{\mathrm{b}} \pm 4.7$ & $57.1^{\mathrm{b}} \pm 7.1$ & $49.9^{\mathrm{b}} \pm 7.8$ & $51.4^{\mathrm{b}} \pm 4.7$ \\
\hline $\mathrm{NI}^{2}$ & $1110^{\mathrm{a}} \pm 72$ & $2895^{\mathrm{b}} \pm 115$ & $4213^{c} \pm 96$ & $4770^{\mathrm{d}} \pm 171$ & $5856^{\mathrm{e}} \pm 109$ \\
\hline $\mathrm{NEX}^{2}$ & $412^{\mathrm{a}} \pm 33$ & $1023^{\mathrm{b}} \pm 59$ & $1781^{\mathrm{c}} \pm 58$ & $2168^{\mathrm{d}} \pm 66$ & $3187^{\mathrm{e}} \pm 44$ \\
\hline $\mathrm{ND}^{2}$ & $697^{\mathrm{a}} \pm 53$ & $1872^{\mathrm{b}} \pm 99$ & $2432^{\mathrm{c}} \pm 43$ & $2602^{c} \pm 183$ & $2669^{c} \pm 114$ \\
\hline \multirow[t]{2}{*}{$b$-value ${ }^{3}$} & $267^{\mathrm{a}} \pm 7$ & $291^{\mathrm{a}} \pm 12$ & $299^{\mathrm{a}} \pm 4$ & $311^{\mathrm{a}} \pm 36$ & $274^{\mathrm{a}} \pm 29$ \\
\hline & \multicolumn{5}{|c|}{ Females $(\mathrm{Na} / \mathrm{Na})$} \\
\hline BW (g) & $246^{\mathrm{a}} \pm 14$ & $425^{\mathrm{b}} \pm 52$ & $555^{\mathrm{b}} \pm 72$ & $544^{\mathrm{b}} \pm 65$ & $478^{b} \pm 58$ \\
\hline DMI (g/d) & $31.2^{\mathrm{a}} \pm 2.1$ & $64.6^{\mathrm{b}} \pm 4.5$ & $68.2^{b} \pm 5.3$ & $65.7^{\mathrm{b}} \pm 4.6$ & $56.7^{\mathrm{b}} \pm 4.6$ \\
\hline $\mathrm{NI}^{2}$ & $1388^{a} \pm 77$ & $3246^{\mathrm{b}} \pm 68$ & $3942^{c} \pm 67$ & $4917^{\mathrm{d}} \pm 86$ & $5659^{\mathrm{e}} \pm 85$ \\
\hline $\mathrm{NEX}^{2}$ & $589^{a} \pm 30$ & $1348^{\mathrm{b}} \pm 51$ & $1752^{\mathrm{c}} \pm 63$ & $2402^{\mathrm{d}} \pm 68$ & $3084^{\mathrm{e}} \pm 108$ \\
\hline $\mathrm{ND}^{2}$ & $799^{a} \pm 54$ & $1898^{b} \pm 47$ & $2190^{\mathrm{bc}} \pm 36$ & $2515^{\mathrm{c}} \pm 35$ & $2575^{\mathrm{c}} \pm 105$ \\
\hline \multirow[t]{2}{*}{$b$-value ${ }^{3}$} & $256^{\mathrm{a}} \pm 5$ & $270^{\mathrm{a}} \pm 7$ & $273^{\mathrm{a}} \pm 7$ & $276^{\mathrm{a}} \pm 5$ & $258^{a} \pm 24$ \\
\hline & \multicolumn{5}{|c|}{ Males $(N a / n a)$} \\
\hline $\mathrm{BW}(\mathrm{g})$ & $185^{\mathrm{a}} \pm 8$ & $321^{\mathrm{ab}} \pm 40$ & $410^{\mathrm{b}} \pm 58$ & $388^{b} \pm 63$ & $430^{\mathrm{b}} \pm 58$ \\
\hline DMI $(g / d)$ & $23.2^{\mathrm{a}} \pm 1.2$ & $49.3^{b} \pm 4.6$ & $58.1^{\mathrm{b}} \pm 7.1$ & $54.0^{\mathrm{b}} \pm 7.1$ & $53.6^{\mathrm{b}} \pm 5.4$ \\
\hline $\mathrm{NI}^{2}$ & $1249^{\mathrm{a}} \pm 54$ & $2970^{\mathrm{b}} \pm 109$ & $4066^{c} \pm 111$ & $5020^{\mathrm{d}} \pm 134$ & $5718^{\mathrm{e}} \pm 95$ \\
\hline $\mathrm{NEX}^{2}$ & $388^{a} \pm 26$ & $891^{\mathrm{b}} \pm 40$ & $1609^{c} \pm 62$ & $2368^{\mathrm{d}} \pm 140$ & $2787^{\mathrm{e}} \pm 109$ \\
\hline $\mathrm{ND}^{2}$ & $862^{\mathrm{a}} \pm 44$ & $2079^{\mathrm{b}} \pm 93$ & $2458^{\mathrm{c}} \pm 56$ & $2652^{\mathrm{cd}} \pm 51$ & $2931^{\mathrm{d}} \pm 80$ \\
\hline \multirow[t]{2}{*}{$b$-value ${ }^{3}$} & $257^{\mathrm{a}} \pm 5$ & $294^{\mathrm{a}} \pm 9$ & $278^{\mathrm{a}} \pm 4$ & $259^{\mathrm{a}} \pm 11$ & $282^{\mathrm{a}} \pm 17$ \\
\hline & \multicolumn{5}{|c|}{ Females $(\mathrm{Na} / \mathrm{na})$} \\
\hline BW (g) & $241^{\mathrm{a}} \pm 18$ & $409^{\mathrm{ab}} \pm 48$ & $494^{b} \pm 63$ & $521^{b} \pm 62$ & $484^{b} \pm 52$ \\
\hline DMI (g/d) & $33.0^{\mathrm{a}} \pm 3.0$ & $61.4^{\mathrm{b}} \pm 4.2$ & $65.1^{\mathrm{b}} \pm 5.0$ & $61.0^{\mathrm{b}} \pm 3.7$ & $56.4^{\mathrm{b}} \pm 3.7$ \\
\hline $\mathrm{NI}^{2}$ & $1483^{a} \pm 98$ & $3162^{\mathrm{b}} \pm 85$ & $4076^{c} \pm 60$ & $4721^{\mathrm{d}} \pm 151$ & $5605^{\mathrm{e}} \pm 98$ \\
\hline $\mathrm{NEX}^{2}$ & $666^{\mathrm{a}} \pm 31$ & $1300^{\mathrm{b}} \pm 64$ & $1867^{\mathrm{c}} \pm 54$ & $2405^{\mathrm{d}} \pm 119$ & $2956^{\mathrm{e}} \pm 95$ \\
\hline $\mathrm{ND}^{2}$ & $817^{\mathrm{a}} \pm 70$ & $1862^{\mathrm{b}} \pm 70$ & $2209^{c} \pm 61$ & $2316^{c} \pm 129$ & $2648^{\mathrm{d}} \pm 45$ \\
\hline$b$-value ${ }^{3}$ & $241^{\mathrm{a}} \pm 3$ & $258^{\mathrm{a}} \pm 9$ & $253^{\mathrm{a}} \pm 9$ & $238^{\mathrm{a}} \pm 17$ & $249^{\mathrm{a}} \pm 8$ \\
\hline
\end{tabular}

$\mathrm{BW}=$ Body weight, $\mathrm{DMI}=$ Dry matter intake, $\mathrm{NI}=\mathrm{N}$ intake, $\mathrm{NEX}=\mathrm{N}$ excretion, $\mathrm{ND}=\mathrm{N}$ deposition (NI-NEX); ${ }^{1}$ LS-Means \pm standard error of means (SEM) according to one-way ANOVA ${ }^{2} \mathrm{mg} / \mathrm{BW}_{\mathrm{kg}}{ }^{0.67}$ per day; ${ }^{3} b$-value: Model parameter indicating the dietary protein quality $\left(b \cdot 10^{6}\right)$;

${ }^{\mathrm{d}}$ LS-Mean values with different superscripts within rows are significantly different $(p<0.05)$

The MBW of naked neck chickens during grower period (days 25-35) depending on diet, genotype and sex was significantly higher in the groups with increased dietary CP levels (Table 5). However, the DMI of homozygous female and heterozygous male chickens was statistically non-significant despite differences in diet. NI and NEX increased significantly from N1-N5 in both the genotypes and sexes. 
Similarly, ND was increased with elevated dietary CP contents. Significantly improved ND values were generally found between diets N1 to N3. As observed in the starter period, the $b$-values of all experimental diets were insignificantly different $(p>0.05)$ independent on varying dietary CP and NI levels for both of genotypes and sexes.

Table 5. Results of $\mathrm{N}$ balance experiments depending on dietary protein content, genotype and sex during grower period (days 25-35) ${ }^{1}$.

\begin{tabular}{|c|c|c|c|c|c|}
\hline \multirow[t]{2}{*}{ Item } & \multicolumn{5}{|c|}{ Diet (Crude Protein as \% of Dry Matter) } \\
\hline & N1 (9.8) & N2 (16.0) & N3 (22.6) & N4 (30.0) & N5 (36.8) \\
\hline & \multicolumn{5}{|c|}{ Males $(\mathrm{Na} / \mathrm{Na})$} \\
\hline BW (g) & $1187^{\mathrm{a}} \pm 45$ & $1519^{\mathrm{abc}} \pm 93$ & $1526^{\mathrm{ac}} \pm 90$ & $1722^{\mathrm{c}} \pm 114$ & $1604^{c} \pm 137$ \\
\hline DMI $(g / d)$ & $98.2^{\mathrm{a}} \pm 6.1$ & $133.2^{\mathrm{b}} \pm 7.0$ & $112.8^{\mathrm{a}} \pm 3.4$ & $116.4^{\mathrm{a}} \pm 5.4$ & $109.7^{\mathrm{a}} \pm 6.7$ \\
\hline $\mathrm{NI}^{2}$ & $1362^{\mathrm{a}} \pm 67$ & $2571^{\mathrm{b}} \pm 41$ & $3091^{\mathrm{c}} \pm 105$ & $3899^{\mathrm{d}} \pm 57$ & $4718^{\mathrm{e}} \pm 100$ \\
\hline $\mathrm{NEX}^{2}$ & $608^{a} \pm 43$ & $1162^{\mathrm{b}} \pm 25$ & $1404^{c} \pm 59$ & $1844^{\mathrm{d}} \pm 77$ & $2606^{\mathrm{e}} \pm 48$ \\
\hline $\mathrm{ND}^{2}$ & $754^{\mathrm{a}} \pm 31$ & $1409^{\mathrm{b}} \pm 40$ & $1688^{c} \pm 72$ & $2055^{\mathrm{d}} \pm 41$ & $2111^{\mathrm{d}} \pm 69$ \\
\hline \multirow[t]{2}{*}{$b$-value ${ }^{3}$} & $288^{\mathrm{a}} \pm 8$ & $282^{\mathrm{a}} \pm 6$ & $295^{\mathrm{a}} \pm 10$ & $315^{\mathrm{b}} \pm 12$ & $273^{\mathrm{a}} \pm 10$ \\
\hline & \multicolumn{5}{|c|}{ Females $(\mathrm{Na} / \mathrm{Na})$} \\
\hline $\mathrm{BW}(\mathrm{g})$ & $1180^{\mathrm{a}} \pm 44$ & $1346^{\mathrm{ab}} \pm 68$ & $1511^{\mathrm{bc}} \pm 88$ & $1544^{\mathrm{bc}} \pm 103$ & $1741^{\mathrm{c}} \pm 79$ \\
\hline DMI (g/d) & $99.5^{\mathrm{a}} \pm 3.5$ & $108.1^{\mathrm{a}} \pm 5.2$ & $112.9^{\mathrm{a}} \pm 2.8$ & $103.9^{\mathrm{a}} \pm 3.0$ & $107.3^{\mathrm{a}} \pm 1.8$ \\
\hline $\mathrm{NI}^{2}$ & $1392^{\mathrm{a}} \pm 39$ & $2265^{\mathrm{b}} \pm 83$ & $3109^{\mathrm{c}} \pm 52$ & $3763^{d} \pm 116$ & $4380^{\mathrm{e}} \pm 112$ \\
\hline $\mathrm{NEX}^{2}$ & $694^{a} \pm 22$ & $1083^{\mathrm{b}} \pm 83$ & $1498^{c} \pm 60$ & $1990^{\mathrm{d}} \pm 70$ & $2565^{\mathrm{e}} \pm 132$ \\
\hline $\mathrm{ND}^{2}$ & $698^{a} \pm 22$ & $1182^{\mathrm{b}} \pm 31$ & $1611^{\mathrm{c}} \pm 45$ & $1773^{\mathrm{c}} \pm 81$ & $1816^{c} \pm 64$ \\
\hline \multirow{2}{*}{$b$-value ${ }^{3}$} & $339^{\mathrm{a}} \pm 5$ & $349^{\mathrm{a}} \pm 15$ & $382^{\mathrm{a}} \pm 16$ & $374^{\mathrm{a}} \pm 22$ & $335^{\mathrm{a}} \pm 23$ \\
\hline & \multicolumn{5}{|c|}{ Males $(\mathrm{Na} / \mathrm{na})$} \\
\hline BW (g) & $1223^{\mathrm{a}} \pm 44$ & $1418^{\mathrm{ab}} \pm 83$ & $1577^{\mathrm{b}} \pm 96$ & $1576^{\mathrm{b}} \pm 166$ & $1704^{b} \pm 93$ \\
\hline DMI (g/d) & $101.0^{\mathrm{a}} \pm 5.2$ & $122.3^{\mathrm{a}} \pm 6.6$ & $121.3^{\mathrm{a}} \pm 4.4$ & $111.7^{\mathrm{a}} \pm 8.6$ & $111.0^{\mathrm{a}} \pm 3.6$ \\
\hline $\mathrm{NI}^{2}$ & $1381^{\mathrm{a}} \pm 65$ & $2471^{b} \pm 64$ & $3245^{c} \pm 67$ & $3967^{d} \pm 92$ & $4593^{e} \pm 53$ \\
\hline $\mathrm{NEX}^{2}$ & $591^{\mathrm{a}} \pm 17$ & $1158^{\mathrm{b}} \pm 45$ & $1431^{\mathrm{c}} \pm 61$ & $1867^{\mathrm{d}} \pm 51$ & $2439^{\mathrm{e}} \pm 54$ \\
\hline $\mathrm{ND}^{2}$ & $790^{\mathrm{a}} \pm 64$ & $1313^{\mathrm{b}} \pm 38$ & $1814^{\mathrm{c}} \pm 35$ & $2100^{c} \pm 45$ & $2154^{\mathrm{c}} \pm 58$ \\
\hline \multirow[t]{2}{*}{$b$-value ${ }^{3}$} & $283^{\mathrm{a}} \pm 7$ & $259^{\mathrm{a}} \pm 6$ & $295^{\mathrm{a}} \pm 8$ & $301^{\mathrm{a}} \pm 5$ & $273^{a} \pm 11$ \\
\hline & \multicolumn{5}{|c|}{ Females $(\mathrm{Na} / \mathrm{na})$} \\
\hline BW (g) & $1138^{\mathrm{a}} \pm 48$ & $1368^{\mathrm{ab}} \pm 68$ & $1513^{\mathrm{b}} \pm 104$ & $1536^{\mathrm{b}} \pm 99$ & $1594^{\mathrm{b}} \pm 74$ \\
\hline DMI $(\mathrm{g} / \mathrm{d})$ & $88.1^{\mathrm{a}} \pm 7.4$ & $116.4^{\mathrm{bc}} \pm 3.7$ & $112.7^{\mathrm{c}} \pm 3.1$ & $105.0^{\mathrm{abc}} \pm 2.5$ & $100.4^{\mathrm{abc}} \pm 2.5$ \\
\hline $\mathrm{NI}^{2}$ & $1255^{\mathrm{a}} \pm 90$ & $2414^{\mathrm{b}} \pm 34$ & $3105^{c} \pm 65$ & $3814^{\mathrm{d}} \pm 149$ & $4346^{\mathrm{e}} \pm 82$ \\
\hline $\mathrm{NEX}^{2}$ & $651^{\mathrm{a}} \pm 26$ & $1187^{\mathrm{b}} \pm 40$ & $1491^{\mathrm{c}} \pm 43$ & $1999^{\mathrm{d}} \pm 82$ & $2490^{\mathrm{e}} \pm 86$ \\
\hline $\mathrm{ND}^{2}$ & $603^{a} \pm 68$ & $1227^{\mathrm{b}} \pm 46$ & $1613^{c} \pm 37$ & $1815^{c} \pm 83$ & $1855^{\mathrm{c}} \pm 43$ \\
\hline$b$-value ${ }^{3}$ & $319^{a} \pm 5$ & $318^{a} \pm 12$ & $350^{\mathrm{a}} \pm 8$ & $346^{\mathrm{a}} \pm 16$ & $314^{\mathrm{a}} \pm 12$ \\
\hline
\end{tabular}

$\mathrm{BW}=$ Body weight, $\mathrm{DMI}=$ Dry matter intake, $\mathrm{NI}=\mathrm{N}$ intake, $\mathrm{NEX}=\mathrm{N}$ excretion, $\mathrm{ND}=\mathrm{N}$ deposition (NI-NEX). ${ }^{1}$ LS-Means \pm standard error of means (SEM) according to one-way ANOVA. ${ }^{2} \mathrm{mg} / \mathrm{BW}_{\mathrm{kg}}{ }^{0.67}$ per day. ${ }^{3} b$-value: Model parameter indicating the dietary protein quality $\left(b 10^{6}\right) .{ }^{a-d}$ LS-Mean values with different superscripts within rows are significantly different $(p<0.05)$.

As expected, the $b$-values (model parameter $b$ multiplied by $10^{6}$ ) calculated according to Equation (3) based on experimental observed ND and derived NMR and $\mathrm{NR}_{\max } \mathrm{T}$ data for corresponding genotypes 
and sexes (Table 6), were not significantly different between graded NI levels, respectively. This current observation gives further support for applications of the model parameter $b$ as an excellent measure of the dietary protein quality $[20,21]$ under conditions of not sufficiently standardized NI.

Table 6. Model parameters for fast growing naked neck chicken related to age period, genotype and sex.

\begin{tabular}{|c|c|c|c|c|c|c|c|c|}
\hline & \multicolumn{4}{|c|}{ Starter period (days 10-20) } & \multicolumn{4}{|c|}{ Grower period (days 25-35) } \\
\hline & \multicolumn{2}{|c|}{$\mathrm{Na} / \mathrm{Na}$} & \multicolumn{2}{|c|}{ Na/na } & \multicolumn{2}{|c|}{$\mathrm{Na} / \mathrm{Na}$} & \multicolumn{2}{|c|}{ Na/na } \\
\hline & Males & Females & Males & Females & Males & Females & Males & Females \\
\hline $\mathrm{NMR}^{1}$ & 262 & 348 & 224 & 392 & 341 & 384 & 346 & 395 \\
\hline $\mathrm{NR}_{\max } \mathrm{T}^{2}$ & 3763 & 3857 & 3965 & 4049 & 3397 & 2881 & 3512 & 3034 \\
\hline $\mathrm{ND}_{\max } \mathrm{T}^{3}$ & 3501 & 3509 & 3741 & 3657 & 3056 & 2497 & 3166 & 2639 \\
\hline \multirow[t]{3}{*}{$\mathrm{PD}_{\max } \mathrm{T}^{4}$} & 13.8 & 13.8 & 14.7 & 14.4 & 25.1 & 20.5 & 26.0 & 21.6 \\
\hline & \multicolumn{8}{|c|}{ Probabilities } \\
\hline & \multicolumn{2}{|c|}{$\mathrm{NMR}^{1}$} & \multicolumn{2}{|c|}{$\mathrm{NR}_{\max } \mathrm{T}^{2}$} & \multicolumn{2}{|c|}{$\mathrm{ND}_{\max } \mathrm{T}^{3}$} & \multicolumn{2}{|c|}{$\mathrm{PD}_{\max } \mathrm{T}^{4}$} \\
\hline Genotype & \multicolumn{2}{|c|}{0.912} & \multicolumn{2}{|c|}{0.626} & \multicolumn{2}{|c|}{0.663} & \multicolumn{2}{|c|}{0.827} \\
\hline Sex & \multicolumn{2}{|c|}{0.035} & \multicolumn{2}{|c|}{0.545} & \multicolumn{2}{|c|}{0.419} & \multicolumn{2}{|c|}{0.572} \\
\hline Age & \multicolumn{2}{|c|}{0.192} & \multicolumn{2}{|c|}{0.005} & \multicolumn{2}{|c|}{0.004} & \multicolumn{2}{|c|}{$<0.001$} \\
\hline
\end{tabular}

\subsection{Nitrogen Maintenance Requirement (NMR)}

Results of the exponential fitting of NEX against NI provide a mathematical approach of the NEX at $\mathrm{N}$ free feeding level (Figure 1). The observed mean daily NMR for male $\left(293 \mathrm{mg} / \mathrm{BW}_{\mathrm{kg}}{ }^{0.67}\right.$ ) and female chickens $\left(380 \mathrm{mg} / \mathrm{BW}_{\mathrm{kg}}{ }^{0.67}\right.$ ) of genotypes under study are summarized in Table 6 . These threshold values are higher than in previous studies with normally feathered counterparts (GRRS [15]: $264 \mathrm{mg} / \mathrm{BW}_{\mathrm{kg}}{ }^{0.67}$; Samadi and Liebert [8]: $252 \mathrm{mg} / \mathrm{BW}_{\mathrm{kg}}{ }^{0.67}$; Pastor et al. [11]: Starter 113, grower $215 \mathrm{mg} / \mathrm{BW}_{\mathrm{kg}}{ }^{0.67}$ ).

In addition, GRRS [15] observed a high variability of NMR data in the literature (172 to 591 $\mathrm{mg} / \mathrm{BW}_{\mathrm{kg}}{ }^{0.67}$ ), depending on genotype, environment and applied design. The statistical analysis yielded a significant effect of sex on NMR. Genotype and age period were identified as non-significant factors of influence (Table 6). Observed NMR data were used for further calculation of $\mathrm{NR}_{\max } \mathrm{T}$. 


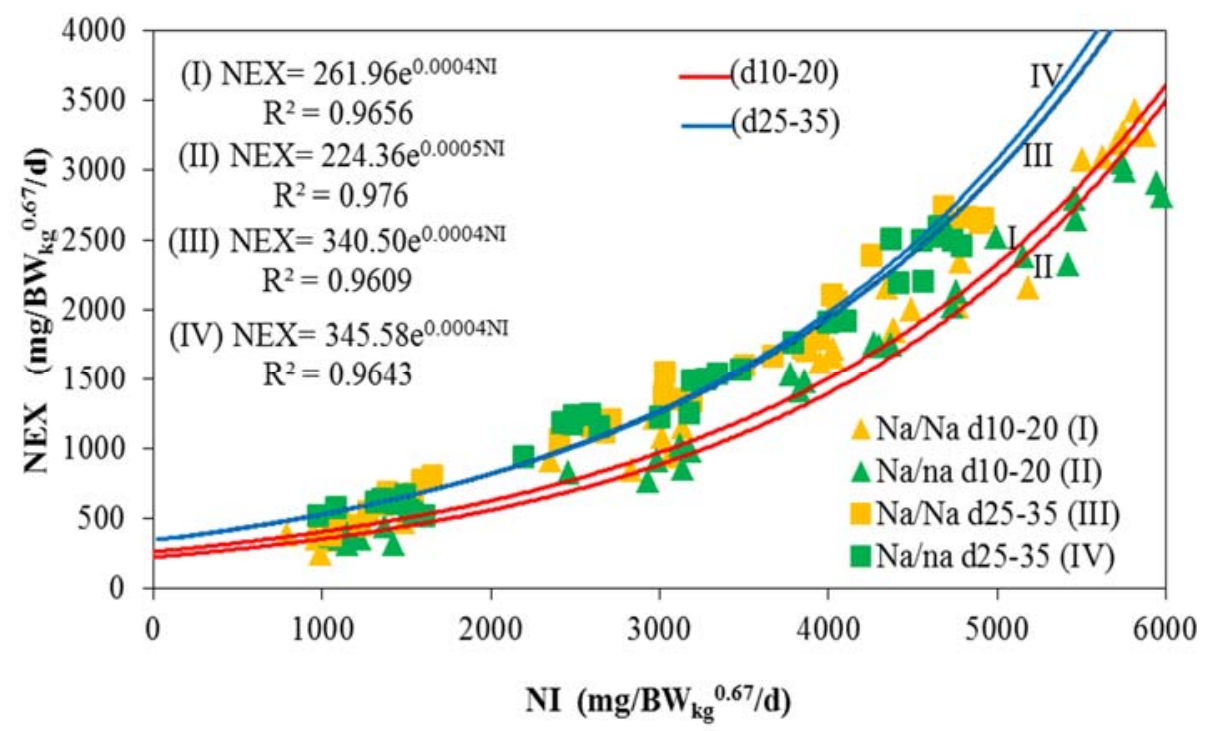

(a)

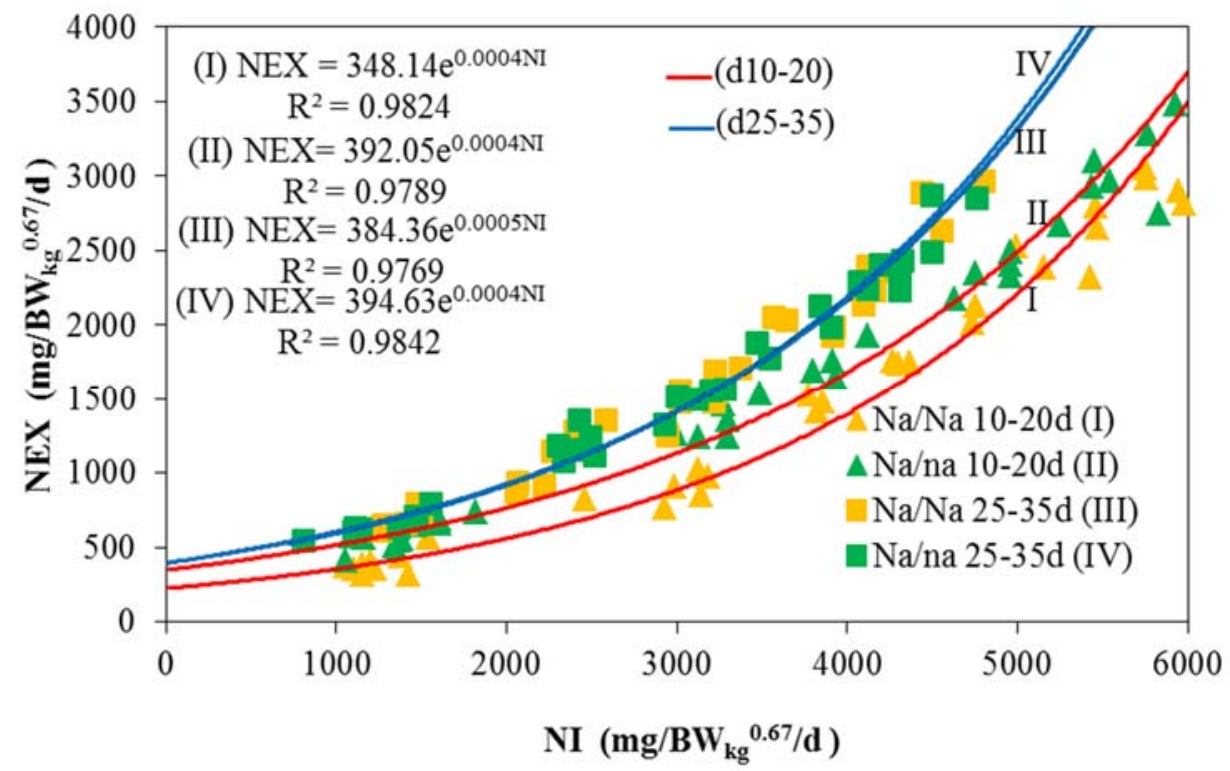

(b)

Figure 1. Estimation of $\mathrm{N}$ maintenance (NMR) by fitting exponential function between daily $\mathrm{N}$ intake (NI) and daily $\mathrm{N}$ excretion (NEX) following graded protein supply depending on sex, genotype and age period. (a) Males; (b) Females.

\subsection{Nitrogen Deposition Potential $\left(N D_{\max } T\right)$}

The threshold values for ND in male and female naked neck growing chickens are demonstrated in Figure 2 and summarized in Table 6. A significant difference was only observed between age periods.

This observed age dependent decline of $\mathrm{ND}_{\max } \mathrm{T}$ is in general agreement with earlier observations [13]. However, results reported from modern broiler genotypes [11] indicate disappearance of this decline of $\mathrm{ND}_{\max } \mathrm{T}$ with increasing age of the birds. In addition, a trend was observed that the heterozygous chicken of both sexes demonstrated a slightly higher threshold value for the theoretical maximum of $\mathrm{ND}\left(\mathrm{ND}_{\max } \mathrm{T}\right)$. This observation should not be over-interpreted from current point of view. 


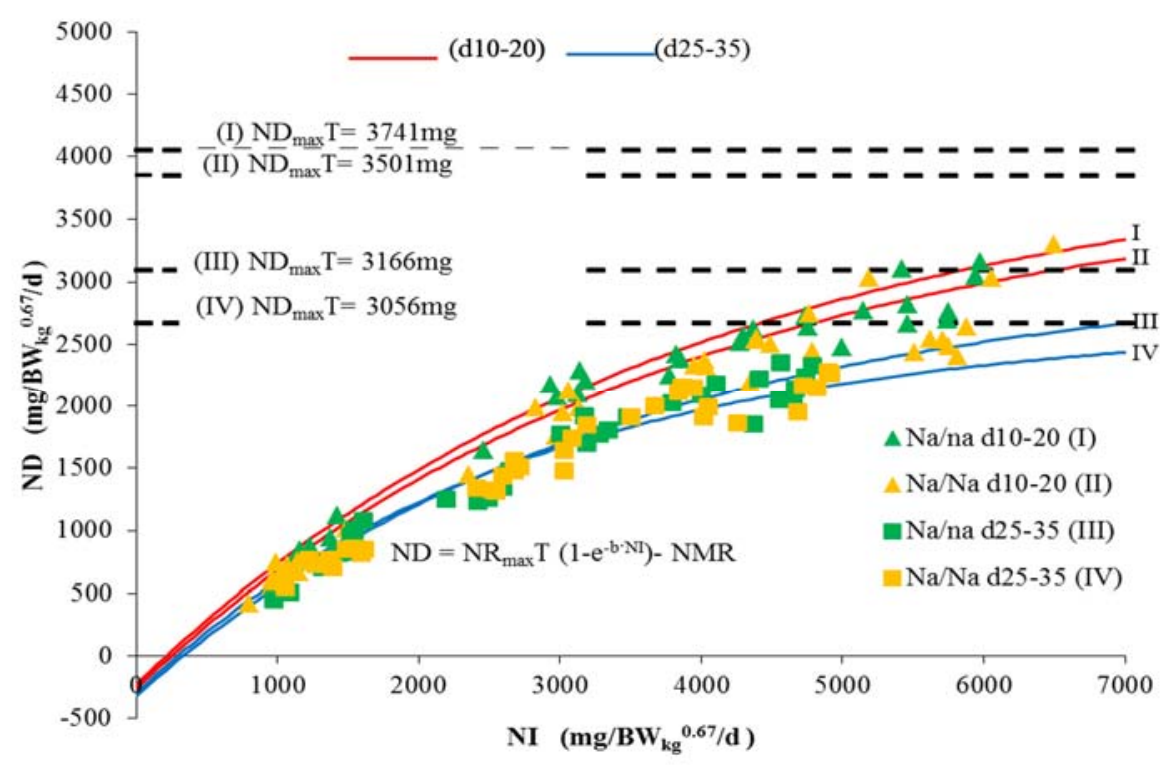

(a)

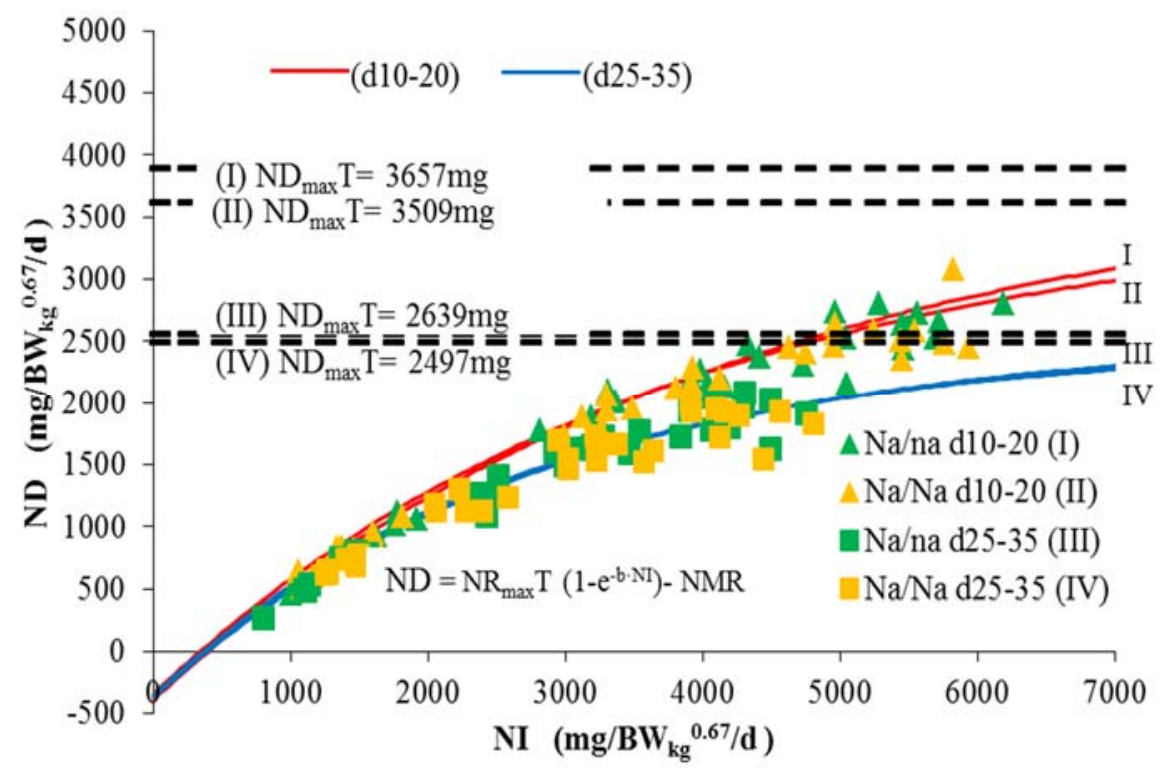

(b)

Figure 2. Estimation of the theoretical potential for daily $\mathrm{N}$ deposition $\left(\mathrm{ND}_{\max } \mathrm{T}\right)$ in naked neck chickens of different sexes, genotypes and age periods based on daily $\mathrm{N}$ deposition (balance) dependent on daily $\mathrm{N}$ intake (NI). $\mathrm{NR}_{\max } \mathrm{T}=$ theoretical maximum for daily $\mathrm{N}$ retention; e = basic number of natural logarithm (ln); $b=$ slope of the N-retention curve (indicating the feed protein quality independent of $\mathrm{NI}$ ); $\mathrm{NMR}=\mathrm{N}$ maintenance requirement.

(a) Males; (b) Females.

\section{Conclusions}

In comparison to commercial breeds of meat type chicken, the naked neck chickens should be better adapted to hot climate conditions. Thus may be advantageous [4] to introduce the naked neck gene into fast growing breeds of meat type chickens. If so, the naked neck genotype could be advantageous 
in developing breeds suitable for environmental conditions with heat stress. However, in addition to improved adaptation to hot climate both acceptable growth performance and efficiency of nutrient utilization are needed. The current study observed that the estimated parameter of theoretical $\mathrm{N}$ deposition potential $\left(\mathrm{ND}_{\max } \mathrm{T}\right)$ of naked neck chickens is slightly below the potential recent commercial genotypes [11] but superior to earlier commercial meat type chickens [13]. From this point of view, meat type chickens of the genotype under study hold an acceptable zoo-technical potential. Accordingly, not acceptable zoo-technical potential of the naked neck genotype are to be expected but acceptable production potential use for efficient broiler production systems. In addition, the current study yielded the first experimental data providing basic parameters $\left(\mathrm{NMR}, \mathrm{NR}_{\max } \mathrm{T}\right)$, which are the precondition for further application of nutritional modeling approaches [21] to derive individual amino acid requirements for the naked neck genotype. Ongoing studies making use of this genotype will help to answer the important question of whether the naked neck gene could modify absolute requirement data for the sulfur containing amino acids methionine and cysteine or the optimal ratio between these amino acids.

\section{Acknowledgments}

The authors are grateful to Aviagen poultry breeders, UK, for the provision of parent stock.

\section{Author Contributions}

Frank Liebert conceived the original idea of the studies. Christian Wecke and Daulat R. Khan designed the experiments. Daulat R. Khan performed the experiments whereas, Christian Wecke supervised it. Ahmad R. Sharifi contributed in raising the breeding stock to get experimental birds, genetic selection of birds and supported statistical analysis of raw data. Daulat R. Khan wrote the first draft of experiment, which was contributed to and subsequently approved by all authors.

\section{Conflicts of Interest}

The authors declare no conflict of interest.

\section{References}

1. Mc Kay, J.C.; Barton, N.F.; Koerhuis, A.N.M.; Mc Adam, J. The challenge of genetic change in the broiler chicken. In The Challenge of Genetic Change in Animal Production; Occasional Publication No. 27. Hill, W.G., Bishop, S.C., Mc Guirk, B., Mc Kay, J.C., Simm, G., Webb, A.J., Eds.; British Society of Animal Science: Edinburgh, UK, 2000; pp. 1-7.

2. Havenstein, G.B.; Ferket, P.R.; Qureshi, M.A. Growth, livability, and feed conversion of 1957 versus 2001 broilers when fed representative 1957 and 2001 broiler diets. Poult. Sci. 2003, 82, 1500-1508. [CrossRef] [PubMed]

3. Janke, O.; Tzschentke, B.; Boerjan, M. Comparative Investigations of Heat Production and Body Temperature in Embryos of Modern Chicken Breeds. Avian Poult. Biol. Rev. 2004, 15, 191-196. [CrossRef] 
4. Garcês, A.; Horst, P. Productive performance of naked neck, frizzle and dwarf laying hens under various natural climates and two nutritional treatments. South Afr. J. Anim. Sci. 2001, 31, 174-180.

5. Yunis, R.; Cahaner, A. The effects of the naked neck $(\mathrm{Na})$ and frizzle $(\mathrm{F})$ genes on growth and meat yield of broilers and their interactions with ambient temperatures and potential growth rate. Poult. Sci. 1999, 78, 1347-1352. [CrossRef] [PubMed]

6. Ajang, O.; Prijono, S.; Smith, W. Effect of dietary protein content on growth and body composition of fast and slow feathering broiler chickens. Br. Poult. Sci. 1993, 34, 73-91. [CrossRef]

7. Yalçin, S.; Özkan, S.; Açikgöz, Z.; Özkan, K. Effect of dietary protein content on live and carcase performance of heterozygous naked neck and normally feathered broilers. Br. Poult. Sci. 1996, 37, 963-969. [CrossRef] [PubMed]

8. Samadi; Liebert, F. Modeling of Threonine Requirement in Fast-Growing Chickens, Depending on Age, Sex, Protein Deposition, and Dietary Threonine Efficiency. Poult. Sci. 2006, 85, 1961-1968.

9. Samadi; Liebert, F. Threonine requirement of slow-growing male chickens depends on age and dietary efficiency of threonine utilization. Poult. Sci. 2007, 86, 1140-1148.

10. Samadi; Liebert, F. Modelling the optimal lysine to threonine ratio in growing chickens depending on age and efficiency of dietary amino acid utilisation. Br. Poult. Sci. 2008, 49, 45-54. [CrossRef] [PubMed]

11. Pastor, A.; Wecke, C.; Liebert, F. Assessing the age-dependent optimal dietary branched-chain amino acid ratio in growing chicken by application of a nonlinear modeling procedure. Poult. Sci. 2013, 92, 3184-3195. [CrossRef] [PubMed]

12. Wecke, C.; Liebert, F. Improving the Reliability of Optimal In-Feed Amino Acid Ratios Based on Individual Amino Acid Efficiency Data from N Balance Studies in Growing Chicken. Animals 2013, 3, 558-573. [CrossRef]

13. Samadi, F.; Liebert, F. Estimation of nitrogen maintenance requirements and potential for nitrogen deposition in fast-growing chickens depending on age and sex. Poult. Sci. 2006, 85, 1421-1429. [CrossRef] [PubMed]

14. NRC (National Research Council). Nutrient Requirements of Poultry, 9th ed.; National Academic Press: Washigton, DC, USA, 1994.

15. GRRS (German Recommendations of Requirement Standards). Empfehlungen zur Energie- und Nährstoffversorgung der Legenhennen und Masthühner (Broiler); Ausschuss für Bedarfsnormen der Gesellschaft für Ernährungsphysiologie, Ed.; DLG-Verlag: Frankfurt, Germany, 1999.

16. WPSA (World's Poultry Science Association). The prediction of apparent metabolizable energy values for poultry in compound feeds. Worlds Poult. Sci. J. 1984, 40, 181-182.

17. Naumann, C.; Bassler, R. VDLUFA-Methodenbuch. Vol. III. Die Chemischen Untersuchungen von Futtermitteln; VDLUFA-Verlag: Darmstadt, Germany, 1997.

18. Thong, H.T.; Liebert, F. Amino acid requirement of growing pigs depending on amino acid efficiency and level of protein deposition. 2nd communication: Threonine. Arch. Anim. Nutr. 2004, 58, 157-168. [CrossRef] [PubMed] 
19. Liebert, F.; Sünder, A.; Mohamed, K. Assessment of nitrogen maintenance requirement and potential for protein deposition in juvenile Tilapia genotypes by application of an exponential nitrogen utilization. Aquaculture 2006, 261, 1346-1355. [CrossRef]

20. Wecke, C.; Liebert, F. Lysine requirement studies in modern genotype barrows dependent on age, protein deposition and dietary lysine efficiency. J. Anim. Physiol. Anim. Nutr. 2009, 93, 295-304. [CrossRef]

21. Liebert, F. Basics and applications of an exponential nitrogen utilization model ("Goettingen approach”) for assessing amino acid requirements in growing pigs and meat type chickens based on dietary amino acid efficiency. In Nutritional Modelling for Pigs and Poultry; Sakomura, N.K., Gous, R., Kyriazakis, I., Hauschild, L., Eds.; CABI Publishing: Wallingford, UK, 2015; pp. 73-87.

(C) 2015 by the authors; licensee MDPI, Basel, Switzerland. This article is an open access article distributed under the terms and conditions of the Creative Commons Attribution license (http://creativecommons.org/licenses/by/4.0/). 